\title{
The essential roles of Mps1 in spermatogenesis and fertility in mice
}

\author{
Qiang Fang $\mathbb{D}^{1}$, Xue-Lin Chen', Lei Zhang ${ }^{1}$, Ya-Bin Li, Tian-Zeng Sun ${ }^{1}$, Chen-Xin Yang ${ }^{1}$, Jian-Feng Chang ${ }^{1}$, \\ Xiao-Mei Yang (1) ${ }^{1}$ and Feng Sun (1)
}

\begin{abstract}
Monopolar spindle 1 (MPS1), which plays a critical role in somatic mitosis, has also been revealed to be essential for meiosis I in oocytes. Spermatogenesis is an important process involving successive mitosis and meiosis, but the function of MPS1 in spermatogenesis remains unclear. Here, we generated Mps1 conditional knockout mice and found that Ddx4-cre-driven loss of Mps 1 in male mice resulted in depletion of undifferentiated spermatogonial cells and subsequently of differentiated spermatogonia and spermatocytes. In addition, Stra8-cre-driven ablation of Mps1 in male mice led to germ cell loss and fertility reduction. Spermatocytes lacking Mps1 have blocked at the zygotene-topachytene transition in the prophase of meiosis I, which may be due to decreased H2B ubiquitination level mediated by MDM2. And the expression of many meiotic genes was decreased, while that of apoptotic genes was increased. Moreover, we also detected increased apoptosis in spermatocytes with Mps1 knockout, which may have been the reason why germ cells were lost. Taken together, our findings indicate that MPS1 is required for mitosis of gonocytes and spermatogonia, differentiation of undifferentiated spermatogonia, and progression of meiosis I in spermatocytes.
\end{abstract}

\section{Introduction}

Spermatogenesis occurs within the testis seminiferous epithelium and consists of the following stages in mice: mitosis of spermatogonia, meiosis of spermatocytes, and maturation of haploid spermatozoa ${ }^{1}$. Initially, spermatogonial stem cells undergo a series of mitosis events and then differentiate into B-type spermatogonia. They then enter meiosis I, becoming primary spermatocytes, as indicated by STRA8 expression ${ }^{2-4}$. Meiosis I involves the stages leptotene, zygotene, pachytene, diplotene, and diakinesis, which can be distinguished by different expression patterns of $\mathrm{SYCP}^{5,6}$. Moreover, at the leptotene stage, double-strand breaks occur and activate $\gamma \mathrm{H} 2 \mathrm{AX}$ and SPO11,8, while at zygonema, RAD51 is highly enriched at the break sites in the chromosome

\footnotetext{
Correspondence: Xiao-Mei Yang (yxm411@tongji.edu.cn) or

Feng Sun (fengsun@outlook.com)

${ }^{1}$ Research Center for Translational Medicine, Shanghai East Hospital, School of Medicine, School of Life Sciences and Technology, Tongji University, Shanghai 200092, China
}

Edited by A. Stephanou strands ${ }^{9}$. During the pachytene stage, meiotic homologous chromosomes combine and form the synaptonemal complex. SYCP3 localizes along the lateral chromosome axis, and SYCP1 loads onto the center to connect the two homologous chromosomes ${ }^{5,6,9}$. Subsequently, diplonema occurs, which features sex bodies marked by $\gamma \mathrm{H} 2 \mathrm{AX}^{10}$, and is followed by diakinesis.

Monopolar spindle 1 (MPS1) is a kinase that is conserved from yeast to mammals that plays a critical role in preventing erroneous chromosome segregation through the spindle assembly checkpoint (SAC) complex $^{11-13}$. During mitosis, MPS1 directly binds to unattached kinetochores through CDC80, after which it phosphorylates and recruits other SAC proteins, such as BUB3, BUBR1, and MAD2, to the kinetochore ${ }^{14-17}$. This process inhibits anaphase-promoting complex/cyclosome (APC/C) activation until all microtubules stably attach to the kinetochores at anaphase onset ${ }^{18}$. MPS1 has important functions not only in mitosis but also in meiosis ${ }^{19}$. In previous research, a mutant mouse strain was generated that expresses a form of $\triangle$ NMPS1 with a 107 -amino acid

\section{(c) The Author(s) 2021}

(c) Open Access This article is licensed under a Creative Commons Attribution 4.0 International License, which permits use, sharing, adaptation, distribution and reproduction cc) in any medium or format, as long as you give appropriate credit to the original author(s) and the source, provide a link to the Creative Commons license, and indicate if changes were made. The images or other third party material in this article are included in the article's Creative Commons license, unless indicated otherwise in a credit line to the material. If material is not included in the article's Creative Commons license and your intended use is not permitted by statutory regulation or exceeds the permitted use, you will need to obtain permission directly from the copyright holder. To view a copy of this license, visit http://creativecommons.org/licenses/by/4.0/. 
deletion at the $\mathrm{N}$-terminus. The $\mathrm{N}$-terminus is involved in binding to the kinetochore ${ }^{20,21}$. Without MPS1 localization, precocious $\mathrm{APC} / \mathrm{C}$ activation leads to the missegregation or nondisjunction events observed in mouse oocytes due to failure of SAC control and chromosome alignment in meiosis $\mathrm{I}^{20}$. Furthermore, MPS1 kinase activation is essential for cohesin protection in a manner dependent on the localization of SGO2 to the centromere in mouse oocytes ${ }^{22}$. However, whether Mps1 plays an essential role in mouse spermatogenesis, especially in mitosis and male meiosis, is still unknown.

To investigate the function of MPS1 in the regulation of spermatogenesis, we generated two conditional Mps1 knockout strains and found that Mps1 is required for mitosis of gonocytes and spermatogonia, differentiation of undifferentiated spermatogonia, and progression of meiosis I in spermatocytes.

\section{Results}

Mps 1 was required for postnatal mitotic divisions of male germ cells

To explore Mps1's function in spermatogenesis, we first characterized the spatiotemporal expression pattern of Mps1 in mice. Quantitative polymerase chain reaction (qPCR) analysis of different adult tissues showed higher Mps1 expression levels in testes than in other tissues (Fig. 1a). In testes, Mps1 expression levels in the mouse testes aged from P3 (postnatal day 3) to P60 showed a dramatic increase from P21, when the male mice started to sexually mature (Fig. 1b). Using the published testes RNA-seq dataset from Soumillon et al. ${ }^{23}$, we further found that Mps1 is especially enriched in spermatocytes (Fig. S1a). All these data suggested that Mps1 may have important roles in spermatogenesis.

For studying the role of Mps1, we generated a conditional Mps1-knockout mouse strain. Specifically, we first generated $M p s 1^{\mathrm{f} / \mathrm{fl}}$ mice by inserting two LoxP sites in intron 5 and intron 8 of the Mps 1 gene, which caused deletion of exons 6-8 and a frameshift mutation driven by Cre recombinase (Fig. S1b). Then, by mating Mps1 floxed mice with $D d x 4$-Cre mice, which specifically express Cre recombinase in germ cells as early as embryonic day 15.5 $(\mathrm{E} 15.5)^{24}$, we obtained germ cell-specific Mps1-knockout $\left(M p s 1^{\mathrm{dKO}}\right)$ mice. Successful knockout was verified by PCR (Fig. S1c, d).

The Mps ${ }^{\mathrm{dKO}}$ mice grew normally like the control mice, and we focused on the spermatogenesis of male mice. First, we examined the morphology of testes from newborn (P0) to adult mice (Fig. 1c). There was no difference between $M p s 1^{\mathrm{dKO}}$ and control mice in terms of testis size at P0. At P10, the testes of $M p s 1^{\mathrm{dKO}}$ and control mice started to become different in size, with the testes of $M p s 1^{\mathrm{dKO}}$ mice being slightly smaller than those of control mice. The difference became much more significant in adult mice. Then, to determine the reason, we assessed the composition of cell types in seminiferous tubules, which are the sites of spermatogenesis (where germ cells develop into spermatozoa). Using hematoxylin-eosin (H\&E) staining, we were able to distinguish two different types of cells on the basis of morphology, i.e., triangle-like Sertoli cells and round germ cells. At P0, both types of cells were visible in $M p s 1^{\mathrm{dKO}}$ and control seminiferous tubules (Fig. 1d). However, there were few germ cells (round-shaped) left in the seminiferous tubules at P10, and no germ cells were present in adult $M p s 1^{\mathrm{dKO}}$ mice; only triangle-like Sertoli cells remained (Fig. 1d). Moreover, many empty seminiferous tubules existed in adult $M p s 1^{\mathrm{dKO}}$ mice. Consistently, no mature sperm were found in the cauda epididymides of $M p s 1^{\mathrm{dKO}}$ mice (Fig. S1e).

To further identify the cell types that disappeared, we employed immunostaining on seminiferous tubule sections of P0 and P10 mice. We used two kinds of marker genes to distinguish cell types: GATA4 for Sertoli cells and DDX4 for germ cells. Consistent with the H\&E staining results, DDX4-positive germ cells and GATA4positive Sertoli cells were present in both control and $M p s 1^{\mathrm{dKO}}$ P0 mice (Fig. 1e). However, almost no DDX4positive germ cells were found in the $M p s 1^{\mathrm{dKO}} \mathrm{P} 10$ testes (Fig. 1f). Nonetheless, Sertoli cells (GATA4-positive) remained unaffected in the $M p s 1^{\mathrm{dKO}}$ testes, as expected. Taken together, the results indicate that Mps1 knockout in early-stage male germ cells results in severe germ cell loss. Previous studies have revealed the important roles of MPS1 in mitosis in somatic cells. Given that germ cells are generated via mitosis of gonocytes and spermatogonia, the findings suggest that the male germ cell depletion in $M p s 1^{\mathrm{dKO}}$ mice might be due to disruption of postnatal mitosis.

\section{Postnatal disruption of Mps 1 led to severe defects in spermatogenesis and significantly reduced male fertility}

Since knocking out Mps1 in Mps $1^{\mathrm{dKO}}$ mouse testes triggered such dramatic germ cell loss that almost no spermatocytes could be observed, the role of MPS1 in the meiosis of spermatocytes remained unclear. To investigate the function of MPS1 in meiosis, we knocked out the Mps1 gene in germ cells by mating Stra8-cre mice, which specifically express Cre recombinase at P3 (early spermatogonia) and exhibit increased expression until P7 (preleptotene spermatocytes) in testes ${ }^{25}$. This strain $\left(M p s 1^{\mathrm{sKO}}\right)$ was verified by PCR (Fig. S2a). The knockout efficiency of Mps1 was confirmed by using Western blotting, which revealed that the protein levels of MPS1 were significantly reduced in the testes of the $M p s 1^{\text {sKO }}$ mice (Fig. S2b).

As we observed, the $M p s 1^{\mathrm{sKO}}$ mice were mostly normal, but their testes were different from those of the control mice. 


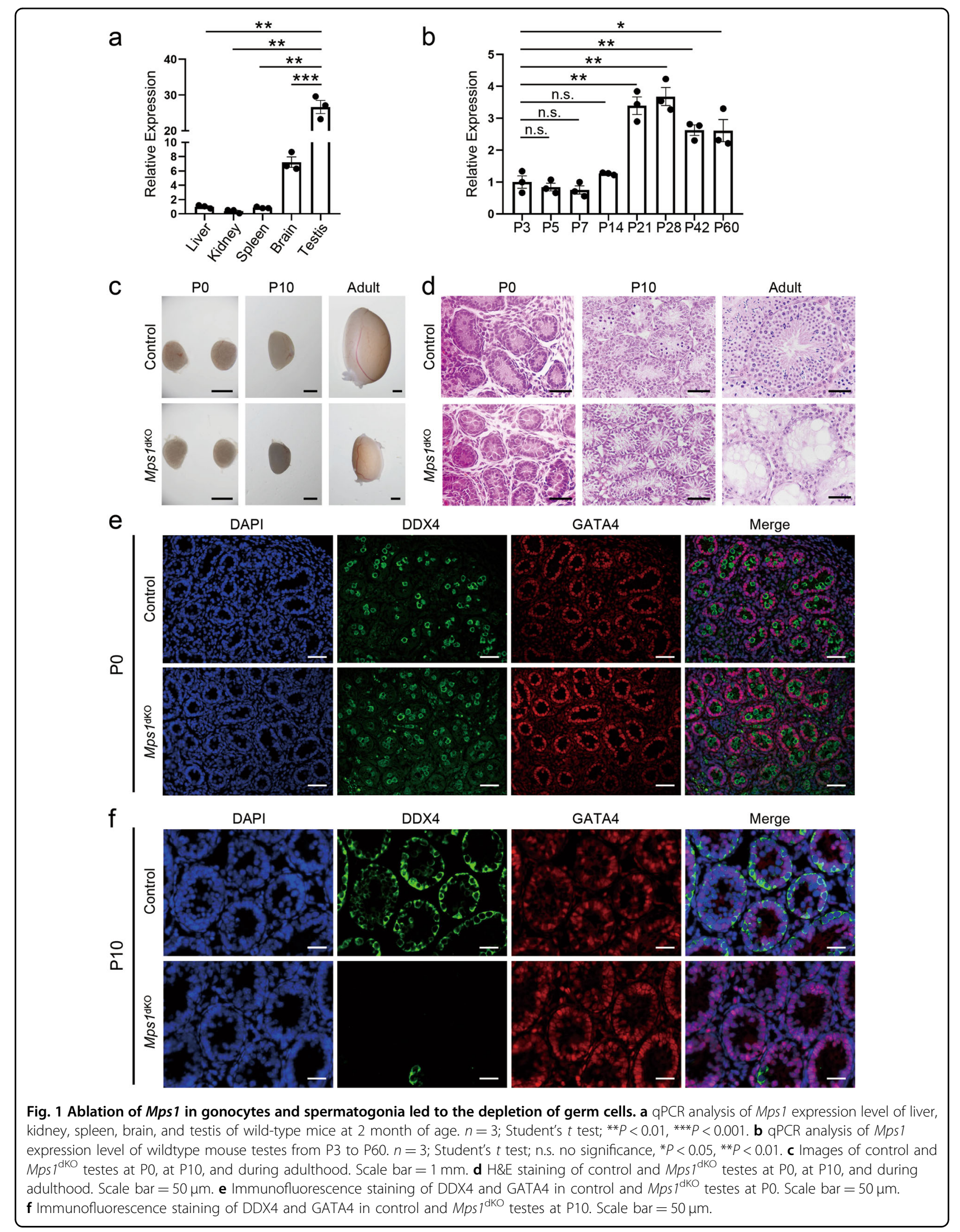


The testes of $M p s 1^{\mathrm{sKO}}$ and control mice were similar in size and mass at P10, but the testes of $M p s 1^{\mathrm{sKO}}$ mice became smaller and lighter than those of control mice at 2 months and 6 months (Fig. 2a, b). Specifically, the testis weight/body weight ratio was significantly reduced from $0.37 \%$ in control mice to $0.12 \%$ in $M p s 1^{\mathrm{sKO}}$ mice at 2 month (32.4\%) and from $0.30 \%$ to $0.08 \%$ at 6 month (28.3\%) (Fig. $2 \mathrm{~b}$ ). In addition, the epididymides were detectably smaller in $M p s 1^{\mathrm{sKO}}$ mice than in control mice (Fig. S2c). Histologically, the seminiferous tubules of $M p s 1^{\text {sKO }}$ mice displayed decreased diameters, reduced cell numbers, and increased empty seminiferous tubule percentages at 2 and 6 months (Fig. 2c, d). The average diameter of $M p s 1^{\mathrm{sKO}}$ seminiferous tubules was reduced to $76 \%$ and $61 \%$ of that of the control mice at 2 and 6 months, respectively (Fig. 2d). At 2 months and 6 months, $35 \%$ and $68 \%$ of $M p s 1^{\text {sKO }}$ seminiferous tubules were degenerated with aberrant spermatogenesis, respectively, while degenerated tubules were hardly detected in the control mice in the time window we examined (Fig. 2d).

Next, we examined whether Stra8-Cre-mediated Mps1 ablation affected sperm production and male fertility. H\&E staining of the cauda epididymis, which is the primary storage site for mature sperm, showed that the density of mature sperm was largely reduced in adult (2 months and 6 months) $M p s 1^{\mathrm{sKO}}$ mice compared to control mice (Fig. 2e). To validate this finding, we released and counted all mature sperm from the cauda epididymides of 6-month-old mice. Strikingly, the average sperm number of $M p s 1^{\mathrm{sKO}}$ mice was decreased to approximately one-tenth of that of control mice (Fig. 2f). We then assessed the fertility of male $M p s 1^{\mathrm{sKO}}$ and control mice. Three-month-old male control and $M p s 1^{\mathrm{sKO}}$ mice were bred with two adult wild-type females for 3 continuous months $(n=5)$. Whereas the control males produced litters of normal size over this period, the $M p s 1^{\mathrm{sKO}}$ males showed significantly reduced fertility. The total number of litters produced by the control mice was 20 (average number of litters: $4 \pm 1.26$ ), and the total number of pups was 141 (average litter size: $7.05 \pm 2.5$ ). However, 3 of 5 $M p s 1^{\mathrm{sKO}}$ mice were infertile during this time period, and another two $M p s 1^{\text {sKO }}$ mice each produced only one litter (average number of litters: $0.4 \pm 0.49$ ); the total pup number dropped to 9 (average litter size: $4.5 \pm 2.27$ ) (Fig. 2 g). Thus, postnatal deletion of Mps1 led to lower productivity of mature sperm and significantly reduced male fertility, suggesting that the reductions in average litter number and size might have been caused by reductions in sperm numbers in the testes of $M p s 1^{\mathrm{sKO}}$ males.

\section{Deletion of Mps1 within differentiating spermatogonia via Stra8-Cre revealed a role for Mps 1 in spermatogonial differentiation}

To determine the mechanism of severe cell loss and fertility reduction in $M p s 1^{\mathrm{sKO}}$ mice, it was necessary to investigate which cell types were lost and when they began to disappear during spermatogenesis. We examined the expression of germ cell markers in testes of mice at P6, P10, and 2 months by immunostaining analysis. The DDX4 staining data showed that the number of $M p s 1^{\text {sKO }}$ mouse germ cells per seminiferous tubule had decreased to $66 \%$ and $57 \%$ of that in control mice by P6 and P10, respectively (Fig. 3a, b), although the testis weight did not differ, as previously mentioned (Fig. 2b). The impact was greater in 2-month-old $M p s 1^{\mathrm{sKO}}$ mice, which retained only $24 \%$ of the germ cells in control mice. On the other hand, the number of PLZF-positive cells per tubule, representing undifferentiated spermatogonia, was the same between $M p s 1^{\mathrm{SKO}}$ mice and controls at different ages, as expected (Fig. 3c, d; Fig. S3a, b). We further investigated the fates of $M p s 1^{\mathrm{sKO}}$ spermatogonia via immunostaining for c-KIT, a marker of differentiated spermatogonia. The results indicated that the c-KITpositive cell number in $M p s 1^{\mathrm{sKO}}$ testes was reduced to $70 \%$ of that in control testes at P6, which suggested that spermatogonial differentiation may have been affected in $M p s 1^{\text {sKO }}$ mice (Fig. 3e, f). In summary, postnatal deletion of Mps1 via Stra8-Cre does not affect the number of undifferentiated spermatogonia but plays a role in spermatogonial differentiation during spermatogenesis.

\section{Loss of Mps 1 compromised the progression of meiosis I and delayed the transition from zygotene to pachytene}

Meiosis is a key feature of spermatogenesis that begins at approximately P10. STRA8 and SYCP3 are a marker of meiosis initiation and a synaptonemal complex component, respectively. Immunostaining analysis showed that the numbers of STRA8-expressing and SYCP3-expressing cells per seminiferous tubule in $M p s 1^{\mathrm{sKO}}$ mice were significantly reduced to approximately $56 \%$ and $45 \%$ of those in control mice at P10. Similarly, the staining results at 2 month revealed that $M p s 1^{\text {sKO }}$ mice had only $30 \%$ of the STRA8-positive and 38\% of the SYCP3-positive cells of control mice (Fig. 4a, b).

According to the spermatocyte composition in the seminiferous tubules, spermatid development in mice has been divided into 12 stages $^{26,27}$. To determine the exact stage of meiosis that was affected by Mps 1 knockout, we performed $\mathrm{H} \& \mathrm{E}$ staining on testes of 6 -month-old mice to trace the seminiferous epithelium stages of the testicular tubules. We found that spermatogenesis seemed delayed at stage IV, during which pachytene spermatocytes should appear and localize inside of the seminiferous lumen. There were fewer pachytene spermatocytes (marked as P) in Mps1-deletion testes than in control testes. Apoptotic pachytenes (marked as AP) were also observed (Fig. 5a). Although some spermatocytes survived in stage IV, fewer round and elongated spermatids formed in $M p s 1^{\text {sKO }}$ testes than in control testes. In addition, meiotic spermatocytes (marked as $\mathrm{M}$ ) were rarely observed in stage XII $M p s 1^{\text {sKO }}$ testes. 

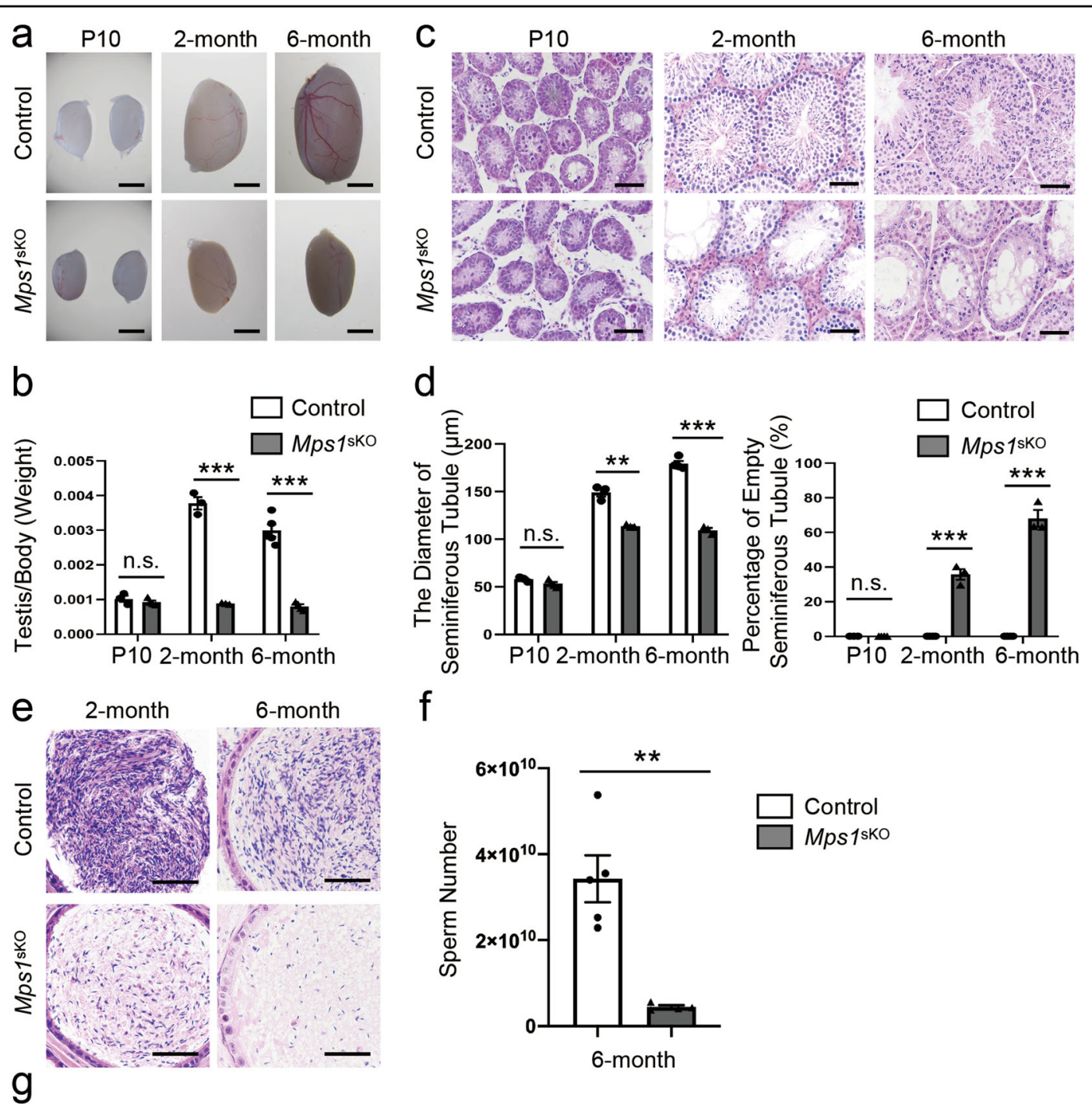

f

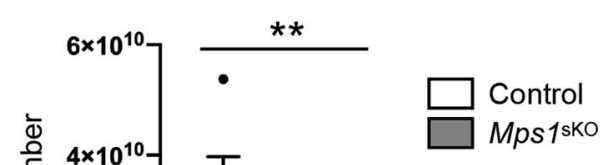

\begin{tabular}{|c|c|c|}
\hline & $\begin{array}{l}\text { Control }(M E A N \pm S E M) \\
(n=5)\end{array}$ & $\begin{array}{l}\text { Mps1sKo }(M E A N \pm S E M) \\
(n=5)\end{array}$ \\
\hline Total litters & 20 & 2 \\
\hline Average litters & $4 \pm 1.26$ & $0.4 \pm 0.48$ \\
\hline Total pups & 141 & 9 \\
\hline Average litter size & $7.05 \pm 2.5$ & $4.5 \pm 2.27$ \\
\hline
\end{tabular}

Fig. 2 Postnatal disruption of Mps 1 led to severe defects in spermatogenesis and significantly reduced male fertility. a Image of control and $M p s 1^{\mathrm{SKO}}$ testes at P10, 2 months of age, and 6 months of age. Scale bar $=2 \mathrm{~mm}$. $\mathbf{b}$ Ratios of testis weight to body weight for control and $\mathrm{Mps} 1^{\mathrm{sKO}}$ mice at P10, 2 months of age, and 6 months of age. $n \geq 3$; Student's $t$ test; n.s. no significance; ${ }^{* * *} P<0.001$. c H\&E staining of control and Mps ${ }^{\text {5KO }}$ testes at P10, 2 months of age and 6 months of age. Scale bar $=50 \mu \mathrm{m}$. d Statistical results for the seminiferous tubule diameters and empty seminiferous tubule percentages of control and $M p s 1^{\text {sKO }}$ testes at P10, 2 months of age, and 6 months of age. At least 100 tubules were counted from at least 3 different mice; Student's $t$ test; ${ }^{* *} P<0.01,{ }^{* * *} P<0.001$. e H\&E staining of control and Mps $1^{\text {5KO }}$ cauda epididymides from 2 and 6 -month-old mice. Scale bar $=50 \mu \mathrm{m}$. $\mathbf{f}$ Sperm counts from the cauda epididymides of at least four different mice at 6-month of age. Student's $t$ test; ${ }^{* *} P<0.01$. g Fertility test of male control and $\mathrm{Mps}^{5 \mathrm{KO}}$ mice. In each group, five 3-month-old male mice were individually mated with two wild-type female mice for 3 continuous months. 

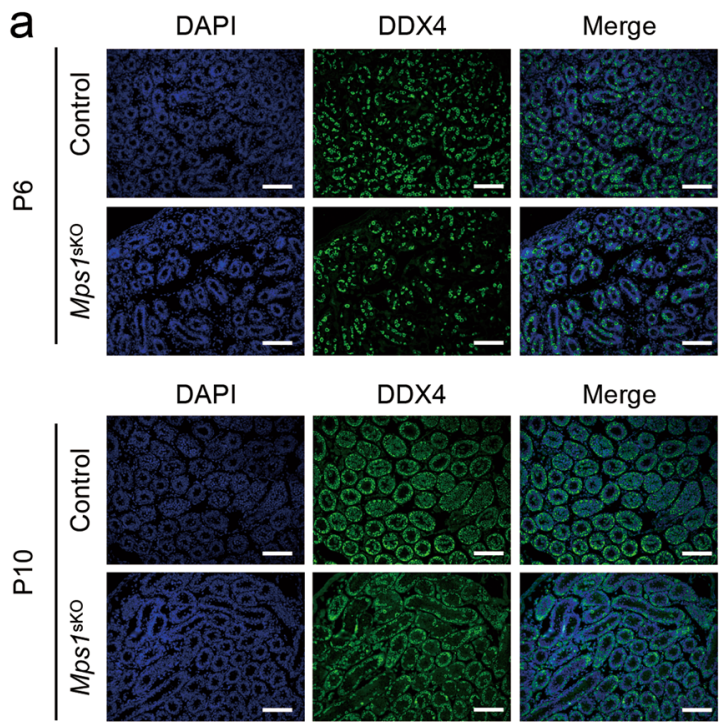

DDX4

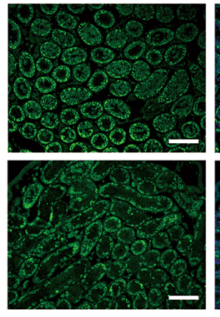

DDX4
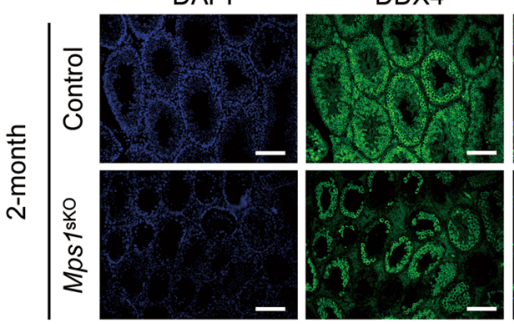

C
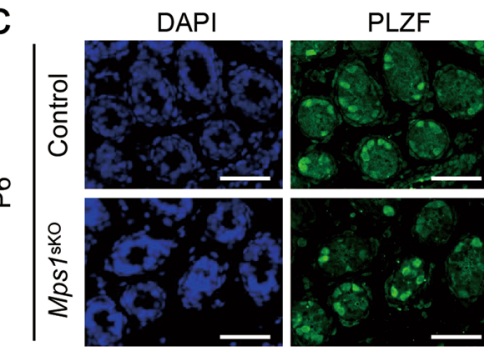

e
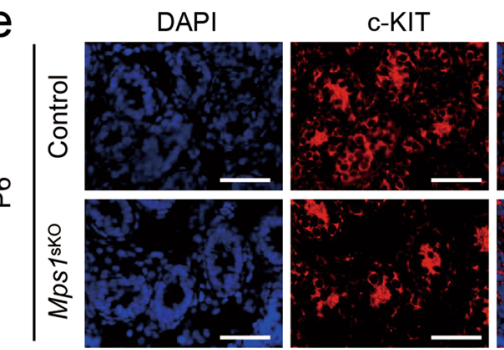

Merge

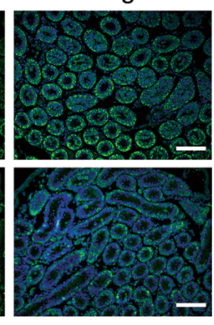

Merge
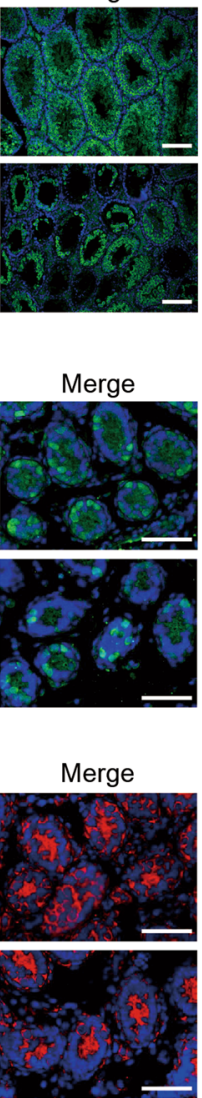
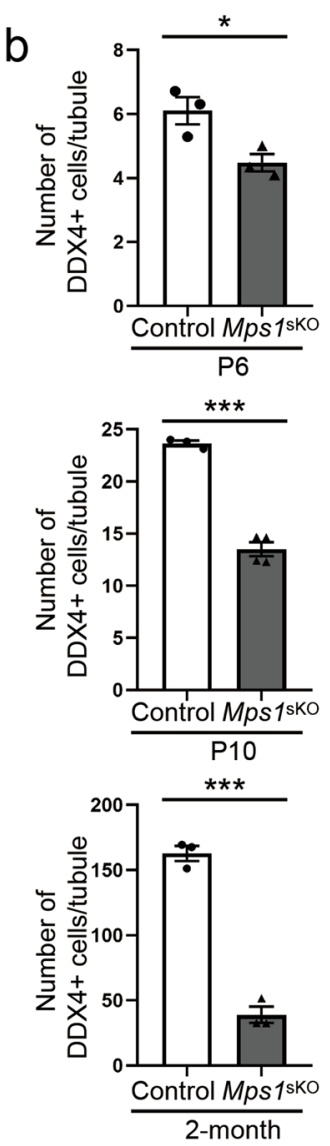

d

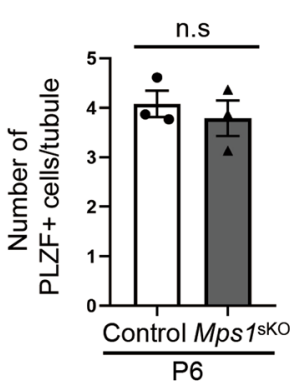

f

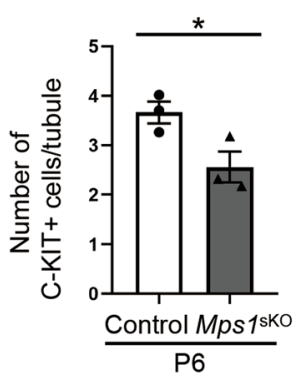

Fig. 3 Deletion of Mps 1 reduced spermatogonial differentiation during spermatogenesis. a Immunofluorescence staining of DDX4 in testes of control and $\mathrm{Mps}^{\mathrm{sKO}}$ mice at P6, P10, and 2 months of age. Scale bar $=50 \mu \mathrm{m}$. $\mathbf{b}$ Statistical results for the number of DDX4-positive cells per tubule at P6, P10, and 2 months of age. $n \geq 3$; Student's $t$ test; ${ }^{*} P<0.05,{ }^{* * *} P<0.001$. c Immunofluorescence staining of PLZF in control and Mps $1^{\text {sKO }}$ testes at P6. Scale bar $=50 \mu \mathrm{m}$. d Statistical results for the number of PLZF-positive cells per tubule at P6. $n \geq 3$; Student's $t$ test; n.s. no significance. e Immunofluorescence staining of c-KIT in control and Mps $1^{\text {sKO }}$ testes at P6. Scale bar $=50 \mu \mathrm{m}$. $\mathbf{f}$ Statistical results for the number of c-KIT-positive cells per tubule at P6. $n \geq 3$; Student's $t$ test; ${ }^{*} P<0.05$. 


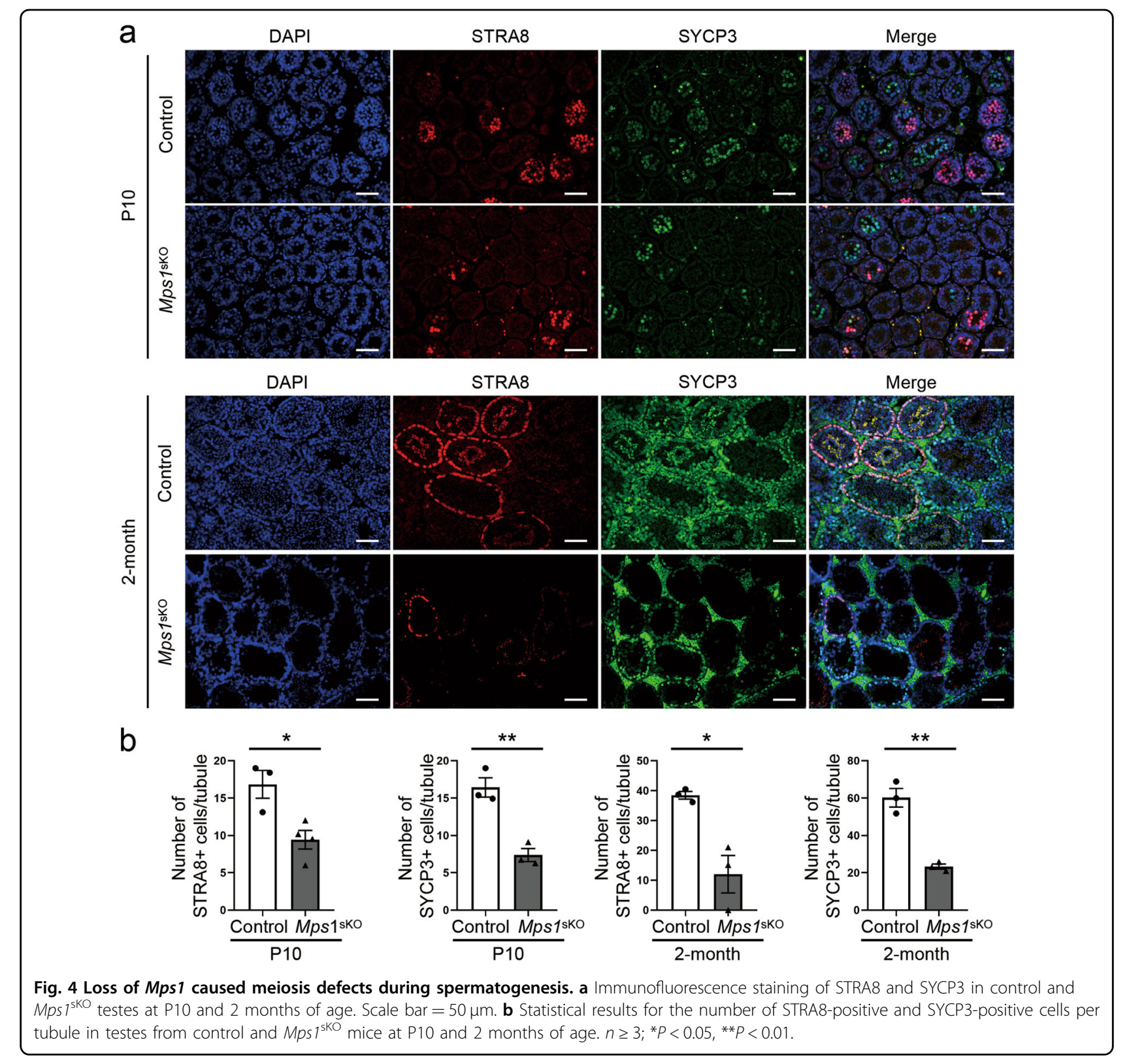

To further identify the delayed stages in the prophase of meiosis, meiotic stage progression was assessed from chromosomal spreads of spermatocytes that were prepared from testes of 1-month male mice and immunostained with anti-SYCP3 and anti- $\gamma \mathrm{H} 2 \mathrm{AX}$ antibodies (Fig. $5 \mathrm{~b}, \mathrm{c})$. In both the $M p s 1^{\mathrm{sKO}}$ and control groups, all five stages from leptotene to diakinesis were observed based on the immunostaining patterns of SYCP3 and $\gamma \mathrm{H} 2 \mathrm{AX}$ in spermatocytes. The proportion of pachytene spermatocytes was reduced from $68.25 \%$ in control mice to $37.85 \%$ in $M p s 1^{\text {sKO }}$ mice, while the proportions of spermatocytes in diplotene and diakinesis were reduced from $8.08 \%$ in control mice to $3.70 \%$ in $M p s 1^{\mathrm{sKO}}$ mice and from $1.56 \%$ in control mice to $0.28 \%$ in $M p s 1^{\mathrm{sKO}}$ mice, respectively. On the other hand, spermatocytes of $M p s 1^{\mathrm{sKO}}$ mice accumulated in leptotene (control mice: 7.24\%; $M p s 1^{\mathrm{sKO}}$ mice: $22.55 \%$ ) and in zygotene (control mice: $14.97 \% ; \mathrm{Mps}^{\mathrm{sKO}}$ mice: $35.61 \%$ ). These data indicated that the transition of zygotene spermatocytes to pachytene spermatocytes was impaired. This finding was investigated further via examination of $\mathrm{H} 3$ core histone phosphorylation at serine 10 (H3pSer10). This type of phosphorylation is coincident with metaphase progression, and the signal then becomes more intense throughout chromatin during the diakinesis phase and the first meiotic metaphase (MI) in spermatocytes $^{28}$. We found that the numbers of H3pSer10-positive germ cells were dramatically reduced in $\mathrm{Mps}^{1}{ }^{\mathrm{sKO}}$ testes (Fig. S4a, b) and that the percentages of H3pSer10- 



d
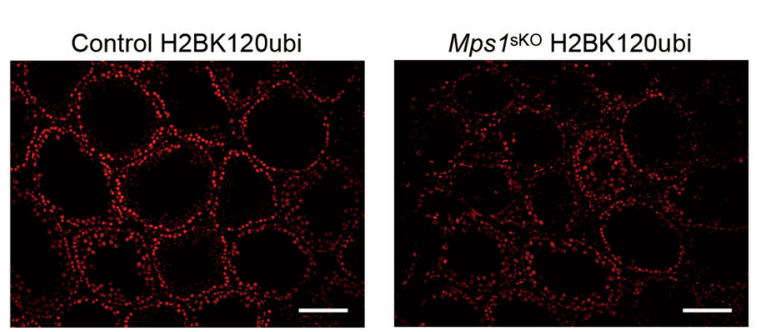

e

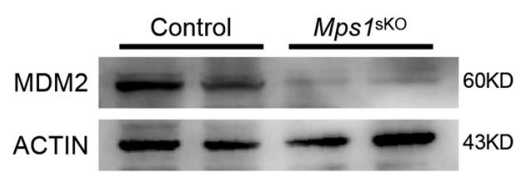

Fig. 5 In $M p s 1^{\text {sko }}$ mouse testes, the zygotene-to-pachytene transition was delayed, and the progression of meiosis I was compromised. a Representative H\&E staining of control and $M p s 1^{\text {SKO }}$ seminiferous tubules. A type A spermatogonia, In intermediate spermatogonia, B type B spermatogonia, P pachytene spermatocytes, L leptotene spermatocytes, Z zygotene spermatocytes, RS round spermatids, ES elongated spermatids, M meiotic divisions, AP apoptosis pachytene, Ser Sertoli. Scale bar $=50 \mu \mathrm{m}$. b Meiotic spermatocyte spreads for control and Mps $1^{\text {sKO }}$ mice at 1 -month of age. Co-staining was performed with SYCP3 and $\mathrm{YH} 2 \mathrm{AX}$ antibodies. Scale bar $=10 \mu \mathrm{m}$. c Percentage of meiotic spermatocytes in each stage. $\mathrm{L}$ leptotene, Z zygotene, P pachytene, D diplotene, Dia diakinesis. A total of 650-1000 spermatocytes were counted from 3 different mice. Student's $t$ test; ${ }^{*} P<0.05$, ${ }^{* *} P<0.01$. d Immunofluorescence staining of H2BK120ubi in control and Mps $1^{\text {5KO }}$ testes at 2 months of age. Scale bar $=100 \mu \mathrm{m}$. e Western blot of MDM2 in control and Mps $1^{5 \mathrm{KO}}$ testes at 2 months of age. 
positive metaphase and anaphase spermatocytes were significantly lower in tubule sections from $M p s 1^{\mathrm{sKO}}$ mice than in those from control mice (Fig. S4b, right). This finding suggested that loss of Mps1 compromised the progression of meiosis I.

Collectively, the evidence indicated that deletion of Mps1 during meiosis resulted in a dramatic decrease in the number of germ cells, which may have been caused by defects in the meiosis process. In particular, the transition from the zygotene to the pachytene stage might have been delayed or partially disrupted in the spermatocytes of $M p s 1^{\text {sKO }}$ mice.

Previously, Xu et al. $^{6}$ found $\mathrm{H} 2 \mathrm{~B}$ ubiquitination regulates the progression of meiosis I by promoting chromatin relaxation, and its E3 ligase Rnf 20 knockout mice had similar phenotypes, such as the smaller size of testes, loss of germ cells and spermatocytes arrested at the prophase of meiosis I. We examined the levels of H2BK120 mono-ubiquitination in the testes of control and $M p s 1^{\mathrm{sKO}}$ mice and noticed that it decreased in $M p s 1^{\text {sKO }}$ testes at 2 months of age compared with that in control testes (Figs. 5d and S4c). MPS1 has been reported to regulate the level of H2BK120ubi mediated by MDM2, another E3 ubiquitin ligase promoting the ubiquitination of $\mathrm{H}_{2} \mathrm{~B}^{28,29}$, and knockdown of Mps 1 by RNAi diminished MDM2 expression in U2OS cells ${ }^{30}$. As expected, the expression level of MDM2 was downregulated in $M p s 1^{\text {SKO }}$ testes, compared to that in control testes (Fig. 5e). In a word, loss of Mps 1 compromised the prophase of meiosis I progress which may be due to decreased H2B ubiquitination level mediated by MDM2.

\section{The expression of meiosis-related genes and apoptosis-} related genes was altered in the testes of $M p s 1^{\text {sKO }}$ mice

To investigated the changes in gene expression after Mps1 deletion, we performed RNA sequencing of P10 testes from control and $M p s 1^{\mathrm{sKO}}$ mice. We found that there were more downregulated genes than upregulated genes in Mps $1^{\mathrm{SKO}}$ mice (Fig. 6a and Table S2). Using cutoffs of a fold change greater than 1.5 and a $P$ value less than $0.01,92$ genes were identified as downregulated, and 24 genes were identified as upregulated (Fig. $6 \mathrm{~b}$ ). We then performed Gene Ontology (GO) analysis to identify the enriched biological processes for each group of differentially expressed genes (DEGs). Notably, for the downregulated genes, the top ten enriched terms were all related to cell division. More importantly, seven of them were meiosis-related, including the meiotic cell cycle, synapsis, and chromosome segregation terms, among others. The most abundant term, the meiotic cell cycle, was enriched for 21 (22.8\%) of the 92 downregulated genes (Fig. 6c and Table S3). To fully discover the biological pathways affected, we relaxed the cut-off for upregulated genes to 1.2-fold, and another 475 genes were identified as upregulated. Of these, 8 out of 189 genes were associated with the apoptosis term. Unsurprisingly, apoptosis stood out as one of the enriched Kyoto Encyclopedia of Genes and Genomes (KEGG) terms for the upregulated genes (Fig. 6d and Table S4). To validate the expression changes in these genes, we examined 13 of them (Mps1, Stra8, Sycp3, Meioc, Sycp1, Syce1, Stag3, Prdm9, Tnf, Capn1, Ptpn13, Sptal, and Tnfrsfla) by qPCR and detected consistent changes in $\mathrm{Mps}^{\text {sKO }}$ testes (Fig. 6e). Therefore, the transcriptomic data indicated that widespread impacts on meiosis and apoptosis resulted from Mps1 deletion.

\section{Mps1 deletion elevated apoptosis in testes}

In order to determine the fates of germ cells with Mps 1 loss, we conducted a TUNEL assay to investigate whether there were more cells undergoing apoptosis in $M p s 1^{\text {sKO }}$ testes than in control testes. On the one hand, there were more seminiferous tubules with TUNEL-positive cells in $M p s 1^{\text {sKO }}$ testes than in control testes (Fig. $6 \mathrm{f}-\mathrm{i}$ ). The percentage of TUNEL-positive tubules increased from $22.8 \%$ in control mice to $28.1 \%$ in $M p s 1^{\mathrm{sKO}}$ mice at $\mathrm{P} 10$ and from $18.1 \%$ to $32.1 \%$ at 2 months. On the other hand, the percentage of TUNEL-positive cells per seminiferous tubule was also increased in the $M p s 1^{\text {sKO }}$ testes (Fig. $6 \mathrm{f}-\mathrm{i}$ ). At P10, on average, $1.06 \%$ of the cells in control seminiferous tubules were TUNEL positive, while $1.39 \%$ of the cells in $M p s 1^{\mathrm{sKO}}$ tubules were TUNEL positive. At 2month of age, the average percentage of TUNEL-positive cells was $0.19 \%$ in control tubules, while $1.60 \%$ in $M p s 1^{\text {sKO }}$ tubules. Overall, the TUNEL assay results showed that many more germ cells in seminiferous tubules underwent apoptosis in $M p s 1^{\mathrm{sKO}}$ mice than in control mice, which indicated that the significant reductions in male germ cell numbers in $M p s 1^{\text {sKO }}$ mice were partly due to increased apoptosis.

\section{Discussion}

MPS1, also known as TTK protein kinase, has been well studied in the context of mitosis in somatic cells ${ }^{14,31}$ and has also been reported to function in meiosis I during oogenesis ${ }^{20}$. Nevertheless, it remains unexplored whether Mps1 plays roles in male spermatogenesis. Here, we explored MPS1 function in mouse spermatogenesis for the first time by utilizing conditional Mps1 knockout in DDX4-positive and STRA8-positive germ cells and demonstrated that MPS1 is critical for spermatogonial differentiation and for the progression of meiosis I spermatocytes. We also revealed that Mps1 may play its role partially by promoting male germ cell apoptosis.

STRA8 expression begins at P3 in the early stage of spermatogonia and lasts through the preleptotene stage ${ }^{25}$. Stra8-Cre mice have been used to investigate gene functions during male meiosis. In our study, we deleted Mps 1 

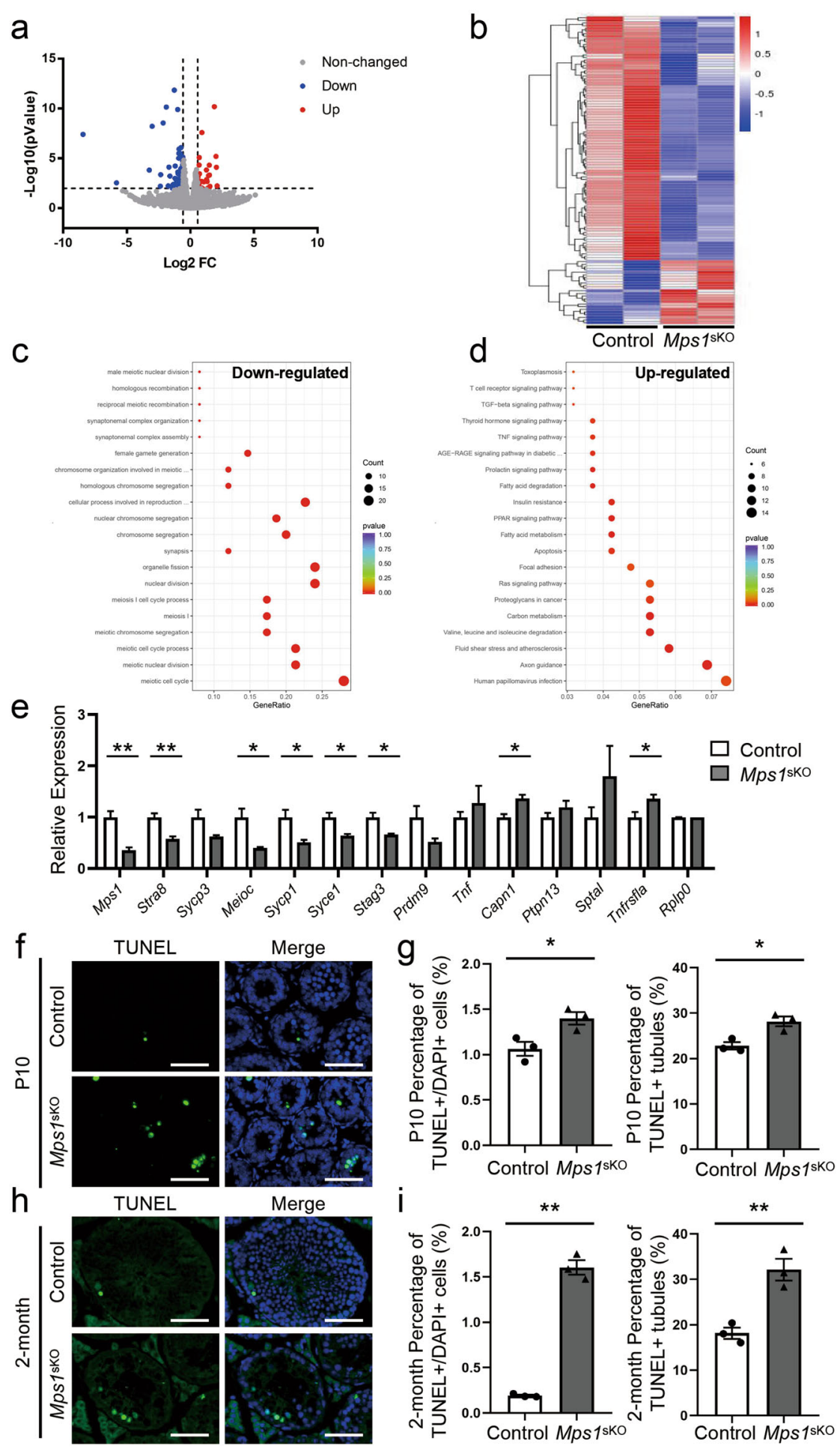

Fig. 6 Loss of Mps 1 altered the expression of genes involved in meiosis and caused abnormal apoptosis in seminiferous tubules. a Gene expression pattern in Mps $1^{\text {sKO }}$ testes compared with control testes at P10. An FC $>1.5$ and a $P$ value $<0.01$ were used as the cut-offs. $\mathbf{b}$ Heatmap of the DEGs between control and Mps ${ }^{5 \mathrm{KO}}$ testes at P10. In total, 92 genes were downregulated, and 24 genes were upregulated. An FC $>1.5$ and a $P$ value $<0.01$ were used as the cut-offs. c Top $20 \mathrm{GO}$ terms for the downregulated DEGs. An FC $>1.5$ and a $P$ value $<0.01$ were used as the cut-offs. $\mathbf{d}$ Top 20 KEGG terms for the upregulated DEGs. An FC $>1.2$ and a $P$ value $<0.05$ were used as the cut-offs. e qPCR analysis of DEGs involved in meiotic biological processes and apoptosis in Mps $7^{\text {5KO }}$ testes at P10. Student's $t$ test; $n=3$; ${ }^{*} P<0.05$. $\mathbf{f}$ Immunofluorescence staining of TUNEL in control and Mps ${ }^{\text {5KO }}$ testes at P10. $\mathbf{g}$ Statistical results for the percentage of TUNEL-positive cells among DAPI-positive cells per tubule and the percentage of TUNEL-positive seminiferous tubules in control and Mps $1^{5 \mathrm{KO}}$ testes at P10. $n \geq 3$; *P $<0.05$. $\mathbf{h}$ Immunofluorescence staining of TUNEL in control and Mps ${ }^{5 \mathrm{KO}}$ testes at 2 months of age. $\mathbf{i}$ Statistical results for the percentage of TUNEL-positive cells among DAPI-positive cells per tubule and the percentage of TUNEL-positive seminiferous tubules in control and Mps ${ }^{\text {5KO }}$ testes at 2 months of age. $n \geq 3 ; * * P<0.01$. 
in STRA8-positive male germ cells and revealed its function in the meiotic cell cycle. However, we also detected germ cell loss at $\mathrm{P} 6$ in $M p s 1^{\mathrm{sKO}}$ seminiferous tubules. Germ cells at this stage included predominantly undifferentiated and differentiated spermatogonial cells that had not entered the meiotic process. Therefore, Stra8-Cre Mps1 knockout affected not only meiosis but also the differentiation of spermatogonial cells. These results suggest that a spermatocyte-specific Cre knock-in mouse line may be more suitable than the knockout mouse line in the current study for research focusing exclusively on meiosis.

Apoptosis is a major form of programmed cell death critical for development and the damage response ${ }^{32}$. Activation of the TNF pathway can induce distinct cellular outcomes, including cell death, inflammation, and cell survival, in a context-dependent manner ${ }^{33}$. In our study, the TNF signaling pathway was upregulated in $M p s 1^{\text {sKO }}$ testes, which might have contributed to the increased apoptosis in $M p s 1^{\text {sKO }}$ testes. Given that loss of Mps1 compromised the progression of meiosis I and increased apoptosis in seminiferous tubules, it seems likely that some germ cells that fail to complete meiosis I may undergo cell death in the absence of MPS1 expression.

Previous studies on Mps1 function in cell division have mainly illustrated its function in the SAC. Hached and his colleagues revealed that Mps1 functions in SAC control and chromosome segregation in oocyte meiosis $\mathrm{I}^{20}$. Our data demonstrate MpsI's role in the prophase of the meiotic cell cycle, showing that Mps1 ablation in this stage results in blockade of the zygotene-to-pachytene transition, which may be due to decreased $\mathrm{H} 2 \mathrm{~B}$ ubiquitination level mediated by MDM2. We also found that the percentages of H3pSer10-positive metaphase and anaphase spermatocytes were significantly decreased in tubule sections from $M p s 1^{\mathrm{sKO}}$ mice, which suggests that Mps1 may play a role in the progression from prophase to metaphase and anaphase. Further studies investigating the underlying mechanisms by which MPS1 plays roles in SAC control and chromosome segregation during male meiosis I are needed to complete our knowledge of MPS1 function in male germ cells.

\section{Materials and methods Animals}

Mps1 floxed conditional knockout mice were generated by targeted homologous recombination (Shanghai Model Organisms). Homology arms, LoxP sites, and selection markers were introduced into the targeting vector via a recombineering-based method. BAC DNA was purchased from the Sanger Institute. Then, the linearized vector was electroporated into ESCs and screened with G418 and Ganc. The correct clones were then injected into C57BL/
6J blastocysts. After mating and identification by PCR with the primers listed below, we obtained Mps1-floxed mice. All mice and samples were selected randomly and proceeded in arbitrary order. No formal randomization techniques were used. All mice used and samples used in IF, H\&E, sequencing analysis were only labeled with mouse ID numbers and no genotypes or treatments were indicated. Genotypes and treatments were labeled after the completion of data acquisition and analysis. The mice were handled according to the university guidelines, and all animal procedures were approved by the Tongji University Institutional Animal Care and Use Program Advisory Committee (protocol number: IACUC-017006).

\section{Genotyping primers}

The primers used to identify the Mps1 floxed genotypes were Mps1-For (forward primer) and Mps1-Re (reverse primer). The floxed band was $353 \mathrm{bp}$, and the wild-type band was $167 \mathrm{bp}$. The primers used to identify the Mps1 deletion alleles were Mps1-min-For and Mps1-Re, which produced a $319 \mathrm{bp}$ band. The band for $D d x 4$ was $240 \mathrm{bp}$, and that for Stra8-Cre was $180 \mathrm{bp}$. The primers for qPCR are listed in Table S1.

\section{Mps1-For: TTGCTTGGTAGTTCTGTGGACT \\ Mps1-Re: AGCAGCTGTAAGTGCAGGAG \\ Mps1-min-For: CTGCGCTAACCTTGTGTTGG \\ Stra8-For: GTGCAAGCTGAACAACAGGA \\ Stra8-Re: AGGGACACAGCATTGGAGTC \\ $D d x 4$-For: CACGTGCAGCCGTTTAAGCCGCGT \\ Ddx4-Re: TTCCCATTCTAAACAACACCCTGAA.}

\section{Histology and immunofluorescence}

For paraffin sectioning, testes were fixed in Bouin's trichrome at $4{ }^{\circ} \mathrm{C}$ overnight. After washing in phosphatebuffered saline (PBS), the testes were dehydrated with gradient concentrations of ethanol and xylene, embedded in paraffin, and cut into $5 \mu \mathrm{m}$-thick sections. H\&E staining was performed and was followed by deparaffinization and rehydration. The slides were imaged with a Nikon microscope.

For frozen sectioning, testes were fixed in 4\% PFA solution at $4{ }^{\circ} \mathrm{C}$ overnight and then dehydrated with $30 \%$ sucrose solution for another 2 days. The testes were dipped in O.C.T. compound and then quickly frozen in liquid nitrogen. Frozen sections with a thickness of $7 \mu \mathrm{m}$ were subjected to heat-induced antigen retrieval (sodium citrate, $\mathrm{pH}=6$ ) in a water bath for $20 \mathrm{~min}$ at $98^{\circ} \mathrm{C}$. The sections were cooled down slowly in retrieval buffer until they reached room temperature. Then, they were blocked and permeated in $5 \%$ bovine serum albumin with $0.2 \%$ Triton in PBS for $1 \mathrm{~h}$ before being incubated with primary antibodies overnight at $4{ }^{\circ} \mathrm{C}$. After washing in PBS three times, the sections were incubated with secondary 
antibodies at room temperature for $2 \mathrm{~h}$. After staining with DAPI (Beyotime) for $5 \mathrm{~min}$, the sections were mounted with a fluorescent mounting medium (Beyotime). All steps were performed with protection from light. Images were obtained with an Olympus microscope and processed with ImageJ and Photoshop software.

\section{Spermatocyte surface spreading}

Testes from 1-month-old mice were subjected to a spermatocyte surface spreading assay as described by the previous research ${ }^{34}$. Briefly, the tunica albuginea was removed, and the tubules were placed into the hypotonic buffer $(30 \mathrm{mM}$ Tris, $50 \mathrm{mM}$ sucrose, $17 \mathrm{mM}$ trisodium citrate dihydrate, $5 \mathrm{mM}$ EDTA, $0.5 \mathrm{mM}$ DTT, and $0.5 \mathrm{mM}$ PMSF, $\mathrm{pH}=8.2$ ) for $30-60 \mathrm{~min}$. Subsequently, the tubules were torn into pieces in sucrose buffer $(100 \mathrm{mM}$ sucrose, $\mathrm{pH}=8.2$ ) and pipetted repeatedly to make a suspension. The cell suspension was smeared onto glass slides containing $1 \%$ PFA and $0.15 \%$ Triton $\mathrm{X}-100, \mathrm{pH}=$ 9.2. The slides were dried overnight in a closed box with high humidity. Finally, the slides were washed twice for 2 min in $0.4 \%$ Photoflo (Kodak) and dried at room temperature. The slides were kept at $-20^{\circ} \mathrm{C}$.

\section{Antibody}

The antibody used in immunofluorescence staining were as follows: anti-DDX4 (ab13840, Abcam), antiGATA4 (ab124265 Abcam), anti-c-KIT (25-1171-82, eBioscience) anti-SYCP3 (ab97672, Abcam), anti-STRA8 (ab49602, Abcam), anti- $\gamma \mathrm{H} 2 \mathrm{AX}$ (ab2893, Abcam), antiH3pSer10 (ab5176, Abcam), anti-MPS1 (ab11108, Abcam), anti-H2BK120ubi (\#5546, CST), anti-MDM2 (sc-965, Santa Cruz), anti H2B (ab1790, Abcam), and anti-ACTIN (sc-47778, Santa Cruz). All fluorophoreconjugated secondary antibodies were obtained from Jackson Immuno Research.

\section{RNA-Seq and analysis}

Total RNA was extracted from whole testes at P10. RNA-Seq libraries were constructed from $1 \mu \mathrm{g}$ of RNA using a NEBNext Ultra ${ }^{\mathrm{TM}}$ RNA Library Prep Kit (NEB, USA) following the manufacturer's instructions. Sequencing was performed on an Illumina NovaSeq platform with $150 \mathrm{bp}$ paired-end reads. DEG analysis was performed with the DESeq2 $\mathrm{R}$ package. GO and KEGG enrichment analysis of the DEGs was implemented with the clusterProfiler $\mathrm{R}$ package. Libraries constructing, sequencing, and data analyzing were performed by Novogene Co., Ltd. (Tianjin, China).

\section{Sperm counting}

Cauda epididymides from 6-month-old mice were removed and cut in $500 \mu \mathrm{l}$ of DMEM. The medium was incubated at $37^{\circ} \mathrm{C}$ for $7 \mathrm{~min}$ to release sperm. After diluting them 50 times, the sperm were analyzed with a cell counter (Countstar).

\section{Assessment of fertility}

To test fertility, 3-month-old conditional knockout and control males were paired with two random adult wildtype females for at least three months. The numbers of offspring from each pregnancy were recorded.

\section{TUNEL assay}

A TUNEL assay was performed following the instructions of an Apoptosis Detection Kit (Millipore). Sections after deparaffinization and rehydration were incubated with proteinase $\mathrm{K}(20 \mu \mathrm{g} / \mathrm{ml})$ for $15 \mathrm{~min}$ and washed 2 times in PBS. Equilibration buffer was immediately applied and kept on the sections for at least $15 \mathrm{~s}$. The excess liquid was gently tapped off, and the workingstrength TDT enzyme was pipetted onto each section. The sections were incubated in a humidified chamber at $37^{\circ} \mathrm{C}$ for $1 \mathrm{~h}$ and then placed in a stop buffer for $10 \mathrm{~min}$. After washing 3 times in PBS, the sections were incubated with an anti-digoxigenin conjugate for $30 \mathrm{~min}$ at room temperature while protected from light and were washed in PBS 3 times again. After staining with DAPI (Beyotime) for $5 \mathrm{~min}$, the sections were mounted with a fluorescent mounting medium. The slides were viewed with a Leica fluorescence microscope.

\section{Statistical analysis}

All statistical data were analyzed by using GraphPad Prism. Sample sizes were described in figure legends. The variances of the two groups were compared by one-way ANOVA, and the $P$ value was determined with a twotailed unpaired Student's $t$ test or with Welch correction. Results were shown as mean \pm s.e.m. Significance was shown as ${ }^{*} P<0.05,{ }^{* *} P<0.01$, ${ }^{* * * *} P<0.001$.

\section{Acknowledgements}

We would like to thank present and past members of the laboratory for their contribution to this study. This research is funded by the National Key R\&D Program of China (2017YFA0103301), and the Fundamental Research Funds for the Central Universities (Tongji University, Grant No. 22120180521).

\section{Author contributions}

Feng Sun and Xiaomei Yang designed the experiments; Fang Qiang performed most of the experiments; Xuelin Chen and Lei Zhang performed part of the experiments. Yabin Li, Chenxin Yang, and Tianzeng Sun performed part of the immunofluorescence experiments. Jianfeng Chang contributed to the data analysis. Feng Sun, Fang Qiang, and Xiaomei Yang wrote the paper. All authors reviewed and approved the paper.

\section{Data availability}

All sequencing data that support the findings of this study have been deposited in the NCBI Gene Expression Omnibus (GEO) under accession number GSE165143.

\section{Ethics approval and consent to participate}

All animal procedures were approved by the Tongji University Institutional Animal Care and Use Program Advisory Committee (Protocol number: IACUC-017-006). 


\section{Conflict of interest}

The authors declare no competing interests.

\section{Publisher's note}

Springer Nature remains neutral with regard to jurisdictional claims in published maps and institutional affiliations.

Supplementary information The online version contains supplementary material available at https://doi.org/10.1038/s41419-021-03815-4.

Received: 4 February 2021 Revised: 5 May 2021 Accepted: 10 May 2021 Published online: 24 May 2021

\section{References}

1. La, H. M. \& Hobbs, R. M. Mechanisms regulating mammalian spermatogenesis and fertility recovery following germ cell depletion. Cell Mol. Life Sci. 76, 4071-4102 (2019).

2. Anderson, E. L. et al. Stra8 and its inducer, retinoic acid, regulate meiotic initiation in both spermatogenesis and oogenesis in mice. Proc. Natl Acad. Sci. USA 105, 14976-14980 (2008).

3. Feng, C. W., Bowles, J. \& Koopman, P. Control of mammalian germ cell entry into meiosis. Mol. Cell Endocrinol. 382, 488-497 (2014).

4. Li, X. et al. The roles of retinoic acid in the differentiation of spermatogonia and spermatogenic disorders. Clin. Chim. Acta 497, 54-60 (2019).

5. $\mathrm{Xu}, \mathrm{K}$. et al. Mettl3-mediated $\mathrm{m}(6) \mathrm{A}$ regulates spermatogonial differentiation and meiosis initiation. Cell Res. 27, 1100-1114 (2017).

6. $\mathrm{Xu}$, Z. et al. $\mathrm{H} 2 \mathrm{~B}$ ubiquitination regulates meiotic recombination by promoting chromatin relaxation. Nucleic Acids Res. 44, 9681-9697 (2016).

7. Keeney, S., Giroux, C. N. \& Kleckner, N. Meiosis-specific DNA double-strand breaks are catalyzed by Spo11, a member of a widely conserved protein family. Cell 88, 375-384 (1997).

8. Jiang, $\mathrm{H}$. et al. MOF influences meiotic expansion of H2AX phosphorylation and spermatogenesis in mice. PLoS Genet. 14, e1007300 (2018).

9. Baudat, F., Imai, Y. \& de Massy, B. Meiotic recombination in mammals: localization and regulation. Nat. Rev. Genet. 14, 794-806 (2013).

10. Fernandez-Capetillo, O. et al. H2AX is required for chromatin remodeling and inactivation of sex chromosomes in male mouse meiosis. Dev. Cell $\mathbf{4}$, 497-508 (2003).

11. Liu, X. \& Winey, M. The MPS1 family of protein kinases. Annu. Rev. Biochem. 81, 561-585 (2012).

12. Manic, G., Corradi, F., Sistigu, A., Siteni, S. \& Vitale, I. Molecular regulation of the spindle assembly checkpoint by kinases and phosphatases. Int. Rev. Cell Mol. Biol. 328, 105-161 (2017).

13. Pachis S. T. \& Kops G. Leader of the SAC: molecular mechanisms of Mps1/TTK regulation in mitosis. Open Biol. 8, 180109 https://doi.org/10.1098/ rsob.180109 (2018).

14. Hiruma, Y. et al. Competition between MPS1 and microtubules at kinetochores regulates spindle checkpoint signaling. Science 348, 1264-1267 (2015).
15. Ji, Z., Gao, H. \& Yu, H. CELL DIVISION CYCLE. Kinetochore attachment sensed by competitive Mps1 and microtubule binding to Ndc80C. Science $\mathbf{3 4 8 ,}$ 1260-1264 (2015).

16. Sun, T. et al. Cellular abundance of Mps1 and the role of its carboxyl terminal tail in substrate recruitment. J. Biol. Chem. 285, 38730-38739 (2010).

17. Sudakin, V., Chan, G. K. \& Yen, T. J. Checkpoint inhibition of the APC/C in HeLa cells is mediated by a complex of BUBR1, BUB3, CDC20, and MAD2. J. Cell Biol. 154, 925-936 (2001).

18. Izawa, D. \& Pines, J. Mad2 and the APC/C compete for the same site on Cdc20 to ensure proper chromosome segregation. J. Cell Biol. 199, 27-37 (2012).

19. Marston, A. L. \& Wassmann, K. Multiple duties for spindle assembly checkpoint kinases in meiosis. Front. Cell Dev. Biol. 5, 109 (2017).

20. Hached, K. et al. Mps1 at kinetochores is essential for female mouse meiosis I. Development 138, 2261-2271 (2011).

21. Wang, W. et al. Structural and mechanistic insights into Mps1 kinase activation. J. Cell Mol. Med. 13, 1679-1694 (2009).

22. El Yakoubi, W. et al. Mps1 kinase-dependent Sgo2 centromere localisation mediates cohesin protection in mouse oocyte meiosis I. Nat. Commun. 8, 694 (2017).

23. Soumillon, M. et al. Cellular source and mechanisms of high transcriptome complexity in the mammalian testis. Cell Rep. 3, 2179-2190 (2013).

24. Gallardo, T., Shirley, L., John, G. B. \& Castrillon, D. H. Generation of a germ cell-specific mouse transgenic Cre line, Vasa-Cre. Genesis 45, 413-417 (2007).

25. Sadate-Ngatchou, P. I., Payne, C. J., Dearth, A. T. \& Braun, R. E. Cre recombinase activity specific to postnatal, premeiotic male germ cells in transgenic mice. Genesis 46, 738-742 (2008).

26. Ahmed, E. A. \& de Rooij, D. G. Staging of mouse seminiferous tubule crosssections. Methods Mol. Biol. 558, 263-277 (2009).

27. Meistrich, M. L. \& Hess, R. A. Assessment of spermatogenesis through staging of seminiferous tubules. Methods Mol. Biol. 927, 299-307 (2013).

28. Minsky, N. \& Oren, M. J. M. C. The RING domain of Mdm2 mediates histone ubiquitylation and transcriptional repression. Mol. Cell. 16, 631-639 (2004).

29. Yu, Z. C., Huang, Y. F. \& Shieh, S. Y. Requirement for human Mps1/TTK in oxidative DNA damage repair and cell survival through MDM2 phosphorylation. Nucleic Acids Res. 44, 1133-1150 (2016).

30. Huang, Y. F., Chang, M. D. \& Shieh, S. Y. TTK/hMps1 mediates the p53dependent postmitotic checkpoint by phosphorylating p53 at Thr18. Mol. Cell Biol. 29, 2935-2944 (2009).

31. Ji, Z., Gao, H. \& Yu, H. Kinetochore attachment sensed by competitive Mps1 and microtubule binding to Ndc80C. Science 348, 1260-1264 (2015).

32. Fuchs, Y. \& Steller, H. Programmed cell death in animal development and disease. Cell 147, 742-758 (2011).

33. Li, M., Sun, S., Priest, J., Bi, X. \& Fan, Y. Characterization of TNF-induced cell death in Drosophila reveals caspase- and JNK-dependent necrosis and its role in tumor suppression. Cell Death Dis. 10, 613 (2019).

34. Peters, A. H., Plug, A. W., van Vugt, M. J. \& de Boer, P. A drying-down technique for the spreading of mammalian meiocytes from the male and female germline. Chromosome Res. 5, 66-68 (1997). 